\title{
Für eine offensive regionale Industriepolitik der IG Metall - Strategien und Maßnahmen
}

FRANK GERLACH, ASTRID ZIEGLER

Die IG Metall -

industriepolitischer Akteur in den Regionen

Industriepolitik findet nicht nur in Brüssel oder Berlin statt. In einem föderalen Staat wie der Bundesrepublik Deutschland haben die Bundesländer sowie die Stadt- und Landkreise Instrumente an der Hand, die Weiterentwicklung von Industrie und wirtschaftsnahen Dienstleistungen zu fördern. Viele Akteure leisten in Regionen mit unterschiedlichen Akzenten einen Beitrag zur Verbesserung der Bedingungen für das gute Gedeihen der Industrie. Deshalb ist gerade die regionale Ebene ein interessantes Feld für neue wirtschafts- und strukturpolitische Umsetzungsstrategien.

In Zukunft werden die Regionen zunehmend gefordert, eigene Antworten auf immer drängendere gesellschaftliche Probleme zu finden. Hierzu zählen die ökologischen Herausforderungen, insbesondere der sich immer deutlicher abzeichnende Klimawandel, die Digitalisierung bzw. die mit Hochdruck vorangetriebene Entwicklung der Künstlichen Intelligenz, die demografische Entwicklung (Überalterung der Gesellschaft) sowie die Globalisierung. Bereits jetzt zeichnen sich dramatische Veränderungen im Bereich der Mobilität sowie der Erzeugung und Verteilung von Energie ab, die sich in den nächsten Jahren noch verstärken werden. Zweifellos werden sie sich auf die industrielle Struktur und Entwicklung sowie die industrienahen Dienstleistungen in den Regionen auswirken. Neben drohenden Gefahren für die Industrie bieten sie auch Chancen für eine positive industrielle Entwicklung. Allerdings sind die Regionen hierbei - trotz eigener Handlungsspielräume - auf die materielle wie immaterielle Unterstützung vonseiten der „höheren“ politischen Ebenen, d.h. der Europäischen Union (EU), des Bundes und der Länder, angewiesen.

Für die IG Metall war die Region schon immer ein wichtiges Handlungsfeld. Sie hat mit eigenen Konzepten und Vorschlägen versucht, auf die industrielle Entwicklung Einfluss zu nehmen und insbesondere eine sich ab- zeichnende De-Industrialisierung in Regionen zu verhindern. Eingebunden in regionale Netzwerke und als starker Partner anerkannt, gingen wichtige industrie- und regionalpolitische Impulse von ihr aus. Die Themenpalette war hierbei ganz unterschiedlich und reichte von Fragen der Industrie- und Wirtschaftsförderung, der Unterstützung von Cluster-, Technologie- und Innovationsinitiativen bis zu Problemen der Infrastruktur, der Arbeitsmarktpolitik sowie der Umweltpolitik. ${ }^{1}$

\section{Industrieller Strukturwandel - eine Konstante in der deutschen Wirtschaftsentwicklung}

Unter dem Strich verlieren in Deutschland etwa eine Million Menschen jedes Jahr ihren Arbeitsplatz durch Stellenabbau und Betriebsschließungen. Dies entspricht etwa $3 \%$ der Beschäftigten. ${ }^{2}$ Davon ist auch die Industrie betroffen. Andererseits entstehen jedoch neue Arbeitsplätze, die in den letzten Jahren rein quantitativ sogar diesen Verlust überwogen. Ob Letzteres in Zukunft so bleiben wird, ist allerdings fraglich, denn die gute wirtschaftliche Entwicklung in Deutschland scheint sich ihrem Ende entgegenzuneigen.

Diese generelle Entwicklung schlug sich ganz unterschiedlich in den Regionen der Bundesrepublik nieder. Ausgenommen die Jahre des sogenannten „Wirtschaftswunders" gab es immer schon Regionen auf der Verliererseite und „Gewinner-Regionen“. Regionen wie Pirmasens, Teile des Ruhrgebiets oder die Südwestpfalz sind als Globalisierungsverlierer geradezu gebeutelt, während andere Gebiete wie z.B. Regensburg oder Landshut als Globa-

\footnotetext{
Vgl. dazu exemplarisch IG Metall Vorstand (Hrsg.) (2014): Industriepolitik heute, Regionale Beispiele der IG Metall, Frankfurt a.M.

2 Vgl. Reissert, B. (2018): Beschäftigtentransfer stärken! Lehren aus einem internationalen Vergleich: Friedrich-Ebert-Stiftung, WISO Diskurs 11/2018, Berlin.
} 
lisierungsgewinner aufgeblüht sind. ${ }^{3}$ Die ostdeutschen Regionen sind heute immer noch mit dem Aufholprozess beschäftigt, der aus den wirtschaftlichen Fehlentwicklungen der DDR-Zeit und der De-Industrialisierung in der Nachwendezeit resultiert. Und nicht zuletzt ist der vom Maschinenbau sowie der Automobilindustrie geprägte Süden Deutschlands nach wie vor Beschäftigungs- und Wohlstandsmotor. Doch auch hier machen sich Sorgen breit, wie es mit der regionalen Entwicklung weitergeht, falls Transformationsprozesse im Bereich der Fahrzeugindustrie voll durchschlagen. Kernregionen der deutschen Industrie könnten unter Umständen in eine Abwärtsspirale geraten. In jedem Fall werden jedoch Arbeitsplätze wegfallen. Selbst wenn es in Zukunft gelingen sollte, neue Arbeitsplätze in ausreichender Zahl zu generieren, wird der nahtlose Übergang in eine neue gleichwertige Beschäftigung absehbar auf große Schwierigkeiten stoßen. Wenn nicht erfolgreich regionalpolitisch gegengesteuert wird, wird sich zwangsläufig die soziale Spaltung in und zwischen den Regionen vertiefen.

\section{Eckpunkte einer regionalen Industriepolitik}

Die Politik steht somit vor großen regional- und industriepolitischen Herausforderungen. Dem will die Bundesregierung laut Koalitionsvertrag durch Einsetzung zweier Kommissionen Rechnung tragen. Die eine mit der Bezeichnung „Wachstum, Strukturwandel und Beschäftigung " hat mittlerweile Vorschläge erarbeitet, die den von der Umsetzung des Klimaschutzplanes betroffenen Regionen konkrete Perspektiven für neue zukunftssichere Arbeitsplätze bieten. Im Prinzip handelt es sich hier um die Gebiete im Rheinland und in Ostdeutschland, die von der Braunkohleförderung und deren energetischer Verwendung abhängig sind. Parallel dazu soll die Kommission „Gleichwertige Lebensverhältnisse“ laut Auftrag Pläne konkretisieren, wie in Zukunft Ressourcen und Möglichkeiten für alle in Deutschland lebenden Menschen gerecht verteilt werden können. Zudem soll ein gesamtdeutsches Fördersystem für alle strukturschwachen Regionen entwickelt werden. Kernelement hierfür soll eine reformierte Gemeinschaftsaufgabe „Verbesserung der regionalen Wirtschaftsstruktur“ (GRW) sein. Es wird sich bald erweisen, ob die hehren Ansprüche der jetzigen Bundesregierung tatsächlich in die Tat umgesetzt werden können. Viel Geld und Zeit ist in jedem Fall erforderlich. Dennoch gilt: Ein neuer regional- und industriepolitischer Anlauf, der den Interessen der Arbeitnehmerinnen und Arbeitnehmer an einer sicheren Beschäftigung auf qualitativ anspruchsvollen, nachhaltigen Arbeitsplätzen in ihren Regionen gerecht wird, ist dringend notwendig. Hierbei sollten aus unserer Sicht Eckpunkte für eine Strategie (Masterplan) sowie für den Einsatz der Instrumente berücksichtigt werden:

(1) Bestandspflege und Weiterentwicklung des Vorhandenen: Keineswegs sollte man sich an das "Alte“ klammern. Strukturkonservatismus ist das Gegenteil einer guten arbeitnehmerorientierten Industriepolitik in den Regionen. Vielmehr sollte der Wandel in den Betrieben durch Weiterentwicklung von Produkten und Innovationen in den Arbeitsprozessen im Interesse der Beschäftigten gestaltet und damit die Arbeitsplätze gesichert werden. Hierbei kann z. B. die IG Metall auf vielfältige Erfahrungen und Erfolge in den Betrieben zurückgreifen, die in ihren unterschiedlichen Publikationen gut dokumentiert worden sind. ${ }^{4}$

(2) Ansiedlung und Entwicklung von Neuem: Die Chancen von Innovationen und neuen technischen Entwicklungen sowie von Marktchancen sollten stärker antizipiert werden. Dies hätte eigentlich ein gutes Management zu leisten. Häufig genug scheitert es an dieser Aufgabe. Zudem handelt es i.d. R. nicht unbedingt im Beschäftigteninteresse. Von den regionalen Akteuren in der Politik ist in dieser Frage wenig zu erwarten, weil ihnen das Know-how fehlt. Deshalb sollten sich die IG Metall und die Betriebsräte gewissermaßen als „Chancenpromotoren“ in der Region und in den Betrieben verstehen, damit die regionale Industrie gestärkt bzw. eine Re-Industrialisierung in der Region erreicht wird. Dabei gilt es, an die vorhandenen betrieblichen Potenziale und das Wissen der Beschäftigten anzuknüpfen und zugleich zu prüfen, welche neuen Möglichkeiten sich in der Forschungs- und Innovationslandschaft für eine Stärkung der Industrie in der Region ergeben. Damit sollen die spezifischen industriellen Zukunftschancen einer Region ermittelt werden.

(3) Rückverlagerung von Produktion: Kostengetriebene Verlagerungen scheitern nicht selten, weil das betriebliche Umfeld am neuen Standort falsch eingeschätzt wird (schlechte Infrastruktur, fehlende betriebliche Netzwerke, fehlende Fachkräfte) und das sowieso schon fragwürdige Kernargument der angeblich zu hohen Lohnkosten in Deutschland angesichts eines immer geringeren Anteils der Lohnkosten an der Wertschöpfung noch fragwürdi-
3 Vgl. Südekum, J./ Dauth, W. / Findeisen S. (2017): Verlierer(-regionen) der Globalisierung in Deutschland: Wer? Warum? Was tun?, in: Wirtschaftsdienst 1/2017, S. 24-31. Viele Regionen waren allerdings in der untersuchten Zeit von 1978 bis 2014 "unauffällig". Ihr Wachstum stand in dem betrachteten Zeitraum im Einklang mit dem westdeutschen Durchschnitt. Dies könnte sich jedoch angesichts der an- stehenden Veränderungen in den kommenden Jahrzehnten ändern.

4 Vgl. dazu exemplarisch: IG Metall Vorstand (Hrsg.) (2015): Industriepolitik und Mitbestimmung. Betriebliche Beispiele der IG Metall, Frankfurt a. M. 
ger geworden ist. ${ }^{5}$ Es gibt somit durchaus Möglichkeiten einer Rückverlagerung von Produktion, die in Deutschland schon vor Jahren aufgegeben wurde. Durch die Digitalisierung, neue Druckverfahren sowie den Einsatz von Robotern rückt eine Produktion „vor Ort“ vielfach wieder in den Bereich des Möglichen.

(4) Bewältigung akuter oder absehbarer Strukturkrisen: Im Fall akuter Strukturkrisen, also wenn die Existenz eines Standortes bzw. ganzer Unternehmen auf dem Spiel steht, gibt es ein bewährtes Krisenmanagementinstrumentarium - wie es vonseiten der IG Metall zuletzt sehr erfolgreich im Fall des Siemenswerkes in Görlitz und weniger erfolgreich im Fall eines Mannheimer Energieanlagenproduzenten von General Electric zum Einsatz kam. Solche „Feuerwehr-Arbeit“ wird im shareholder-getriebenen Kapitalismus immer wieder die IG Metall in den Regionen fordern und muss damit notwendigerweise im Sinne der Vorbereitung auf den „Ernstfall“ ein genuiner Bestandteil eines regionalen Masterplans sein.

Selbstverständlich muss eine solche regionale Strategie in eine generelle industriepolitische Strategie auf EU-, Bundes- sowie Bundesländer-Ebene eingebettet sein.

\section{Instrumente einer regionalen Industriepolitik}

In Deutschland wurden und werden staatlicherseits viele Instrumente bzw. Förderprogramme - wie die GRW, EU-Strukturfonds, Projekt- bzw. Forschungsförderung, Qualifizierungsmaßnahmen - entwickelt und eingesetzt, um die Wettbewerbschancen von Regionen zu verbessern. Wenn ein Unternehmen z.B. ein neues Werk errichten will, dann setzt geradezu ein Wettlauf der Regionen ein, um es für die eigene Region zu gewinnen. Dem Investor wird gewissermaßen der rote Teppich ausgerollt. Subventionierung von Grund und Boden, monetäre Zuschüsse für die Investitionen, Qualifizierungsmaßnahmen für potenziell Beschäftigte - alles dies und vieles mehr gehört in das Repertoire einer regionalen Industriepolitik.

Die eigenen Handlungsspielräume von Kommunen und Landkreisen bei der Auswahl der Instrumente sind jedoch begrenzt. Die Variation der Hebesätze der Gewerbesteuer, die Flächennutzungsplanung, die kommunale Wohnungsbaupolitik, der Bau von Bildungseinrichtungen und Kitas sowie der Straßenbau gehören wesentlich dazu. Damit haben sie zwar nicht unwichtige Hebel in der Hand, sie reichen jedoch für eine wirkungsvolle regionale Industriepolitik nicht aus. In der Regel nutzen sie das Instrumentarium, das ihnen die Bundesländer, der Bund und die EU zur Verfügung stellen. Denn die Entscheidungen über die Bereitstellung zentraler Instrumente der direkten und indirekten Wirtschaftsförderung werden auf diesen Ebenen gefällt. Regionen sind somit gewissermaßen „Nachfrager“, wenn es sich um den Einsatz von Mitteln für die regionale Industriepolitik handelt. Sie können allerdings im politischen bargaining auf die Ausgestaltung der Instrumente vornehmlich in den Bundesländern, aber auch im Bund, weniger im Bereich der EU Einfluss nehmen. Diese Entscheidungsstrukturen müssen bei der Konzipierung einer regionalen Industriepolitik immer im Auge behalten werden

Wenn Maßnahmen - wie die Subventionierung eines neuen Industriebetriebes - dazu dienen, die Chancen strukturell benachteiligter Regionen auf industrielle Investitionen und Neuansiedlungen zu verbessern, so können sie im Sinne des regionalen Ausgleichzieles fallweise unterstützt werden. Allerdings handelt es sich hierbei um eine Gratwanderung. Die Erfahrungen aus der Vergangenheit zeigen: Häufig werden Subventionen „abgegriffen“, obwohl die Investition sowieso getätigt worden wäre (Mitnahmeeffekte) bzw. sie sich nach kurzer Zeit als obsolet erweist - wie im Fall des Nokia-Werkes in Bochum geschehen. Deswegen sollte die regionale Förderkulisse im Interesse der Arbeitnehmerinnen und Arbeitnehmer reformiert werden. Folgende Prinzipien sind hierbei zu berücksichtigen:

(1) Die Förderungspolitik sollte sich weniger auf direkte Subventionen für Unternehmen als vielmehr auf das wettbewerbliche Vorfeld konzentrieren. Noch intensiver als in der Vergangenheit sollte bei direkter Subventionierung auf die Verhinderung von Mitnahmeeffekten geachtet werden. Die stärkere Einbeziehung von Betriebsräten und Gewerkschaften im Prozess der Bewilligung und Realisierung von Förderanträgen wäre hierfür ein Weg.

(2) Die Regionen sollten durch eine gute Lebensqualität, ein gutes Angebot an bezahlbaren Wohnungen, ein hervorragendes Bildungsangebot, einen gut qualifizierten Fachkräftebestand, eine exzellente Verkehrsinfrastruktur für die in der Region angesiedelten Unternehmen sowie für neue Industriebetriebe attraktiver gemacht werden. In dieser Hinsicht ist infolge der Sparpolitik in vielen Regionen in den letzten Jahren vieles sträflich vernachlässigt worden. Insbesondere die Befähigung zur Beschäftigung sollte im Vordergrund stehen. Hierzu sind die vorhandenen Instrumente der Förderpolitik sowie der Bundesagentur für Arbeit anzuwenden und auszubauen. Das gleiche gilt für die betriebliche Weiterbildung und Qualifikation.

(3) Zugleich sollte die Kooperation zwischen Unternehmen, Forschungseinrichtungen und Verbänden stärker als in der Vergangenheit gefördert werden. Aus vielen

5 Hierfür gibt es viele Beispiele, wie etwa die empirischen Untersuchungen von Steffen Kinkel und des Fraunhofer ISI Karlsruhe gezeigt haben. Vgl. z. B.: Digitalisierung bringt Produktion zurück an den Standort Deutschland, Studie von Hochschule Karlsruhe und Fraunhofer ISI für den VDI, https://www.hs-karlsruhe.de/press/digitalisierung-bringt-produktion-zurück 
Untersuchungen ist bekannt, dass Netzwerke und Cluster für eine positive regionale industrielle Entwicklung (inkl. der industrienahen Dienstleistungen) unverzichtbar sind. Eine Förderpolitik, die jenseits des Marktes ansetzt, kann somit die Unternehmen stärken. Anders als in der Vergangenheit sollten auch hier die Mitwirkungsrechte der Gewerkschaften und der Betriebsräte gestärkt werden.

(4) Ein Paradigmenwechsel der Rolle des Staates als Treiber von Innovationen und Gestalter neuer Märkte ist u.E. für die industriepolitischen Gestaltungsmöglichkeiten in den Regionen notwendig. Mazzucato wie auch Block/ Keller ${ }^{6}$ haben anhand vieler Beispiele nachgewiesen, in welchem Ausmaß staatlich angestoßene Innovationen bei technologischen Sprüngen zur Entstehung neuer Produkte und damit neuer Märkte geführt haben. Beispielsweise stand die National Science Foundation (NSF) hinter dem Algorithmus von Google und wesentliche Technologien des iPhones (Internet, GPS, touch-screen display und SIRI) wurden von staatlichen Einrichtungen in den USA direkt finanziert. Insofern ist es nur konsequent, nicht nur die Risiken solcher Forschungsvorhaben zu sozialisieren, sondern den Staat ganz konkret an den Gewinnen teilhaben zu lassen und zudem dort die Produkte zu produzieren, wo die Innovationen staatlicherseits mit viel Geld generiert worden sind.

(5) Bei der Wirtschaftsförderung ist auf die Umsetzung von "guter Arbeit“" zu achten. Hierbei gibt es in einigen Bundesländern bereits jetzt schon Regelungen, deren Übertragung auf andere Bundesländer, andere Förderprogramme sowie deren Erweiterung notwendig ist. In den Betrieben sollten jedoch die Gewerkschaften mit den Betriebsräten prüfen, ob die Regeln bei der Vergabe von Fördermitteln adäquat umgesetzt werden.

(6) Forschung und Entwicklung ist auch in den Industriebetrieben sowie in den staatlichen und außerbetrieblichen Forschungseinrichtungen in den Regionen ein Megathema. Wenn die Wirtschaftsministerin des Landes Baden-Württemberg lapidar feststellt, dass die Innovationsbereitschaft und die Innovationsdynamik vor allem bei den KMU in Baden-Württemberg nachgelassen haben, dann sollten eigentlich die Alarmglocken läuten. ${ }^{7}$ Auf diese Weise können die zukünftigen oben skizzierten

6 Vgl. Mazzucato, M. (2013): The entrepreneurial state: Debunking the public vs. privat myth in risk and innovation, London; Block, F./ Keller, M. R. (2011): State of innovation the U.S. Government's role in technology development, Boulder.

7 Vgl. Hoffmeister-Kraut, N. (2017): Industrie in Baden-Württemberg: Pfeiler für Beschäftigung, in: Lemb, W. (Hrsg.): Industriepolitik in den Bundesländern Perspektiven, Maßnahmen, Ziele, Frankfurt a. M.
Anforderungen nicht gemeistert werden. Auch die IG Metall und die Betriebsräte sind hier gefordert. Sie müssen prüfen, ob die Unternehmen diese Aufgaben in dem gebotenen Tempo forcieren, und dafür sorgen, dass die Interessen der Beschäftigten auf Weiterqualifikation und Beschäftigung gewahrt bleiben.

(7) Ein weiteres Megathema in der Förderpolitik ist die Nachhaltigkeit. Energie- und Ressourceneffizienz sowie die Entwicklung nachhaltiger Produkte sind mehr denn je in der Förderpolitik sowie in den Betrieben umzusetzen. Staatliche Programme können hierbei hilfreich sein. Zugleich eröffnen sich hierdurch neue Beschäftigungschancen und Möglichkeiten für eine Zukunftssicherung der regionalen Industrie. Die bisherige Bilanz ist gemischt. Im Fall der stark subventionierten Solarindustrie gab es ein kostenträchtiges Debakel. Hingegen erlebte die Windkraftindustrie in den vergangenen Jahren einen Aufschwung, jedoch gefährden nun drohende Betriebsschließungen und Entlassungen die Zukunft dieser für Deutschland außerordentlich wichtigen Branche.

(8) Last but not least können öffentlich geförderte, arbeitsorientierte regionale Analysen helfen, regionale Defizite $\mathrm{zu}$ benennen und entsprechende Maßnahmen in der Region wie auch in den Betrieben einzufordern. Zudem können sie Schwachstellen des Wissenstransfers von den außerbetrieblichen Forschungs- und Entwicklungseinrichtungen und den Hochschulen in die regionalen Betriebe ermitteln. Hier sind auch die Stiftungen der deutschen Gewerkschaften in die Pflicht zu nehmen.

Zusammenfassend kann gesagt werden, dass angesichts neuer Entwicklungen in den Regionen und damit neuer Chancen und Risiken der Weg von einer reaktiven zu einer offensiven Regional- und Industriepolitik noch stärker zu forcieren ist. Wie in der Vergangenheit kann und muss die IG Metall im Interesse der Arbeitnehmerinnen und Arbeitnehmer hierbei ein Treiber sein.

\section{AUTOREN}

FRANK GERLACH, Dr., war bis 2009 in der Forschungsförderung der Hans-Böckler-Stiftung für den Bereich „Strukturwandel - Innovation und Beschäftigung“ zuständig. Arbeitsschwerpunkt: Industrie- und Innovationspolitik, Regional- und Strukturpolitik

fk-gerlach@t-online.de

ASTRID ZIEGLER, Dr., ist Ressortleiterin für Industrie-, Struktur- und Energiepolitik beim Vorstand der IG Metall und dort zuständig für Fragen der Industriepolitik und des industriellen Strukturwandels in den Branchen der Metall- und Elektroindustrie.

astrid.ziegler@igmetall.de 